\title{
PERSPECTIVE
}

\section{Would You Be Surprised If This Patient Died This Year? Advance Care Planning in Substance Use Disorders}

Michelle J. Fleshner, MD, MPH' , Amy J. Kennedy, MD' ${ }^{7}$, Peter J. Veldkamp, $M D, M S^{2}$, and Julie W. Childers, MD, $M S^{7,3}$

'Division of General Internal Medicine, University of Pittsburgh Medical Center, Pittsburgh, USA; ${ }^{2}$ Division of Infectious Diseases, University of Pittsburgh Medical Center, Pittsburgh, USA; ${ }^{3}$ Section of Palliative Care and Medical Ethics, University of Pittsburgh Medical Center, Pittsburgh, USA.

Primary care physicians are increasingly incorporating screening tools for substance use disorders (SUDs) and referral to treatment into their practice. Despite efforts to provide access to treatment, patients with SUDs remain at an increased risk of mortality, both from overdose and from general medical conditions. Advance care planning (ACP) is recommended for patients with chronic, progressive medical conditions such as malignancies or heart failure. Though SUDs are widely acknowledged to be chronic diseases associated with an increased risk of mortality, there has been little discussion on ACP in this population. ACP is a discussion regarding future care, often including selection of a surrogate decision-maker and completion of an advanced directive. ACP has been associated with better quality of end-of-life and care more consistent with patient preferences. Studies in other vulnerable populations have shown that marginalized and high-risk individuals may be less likely to receive ACP. Similarly, patients with SUDs may employ different decision-makers than that defined by law (i.e., friend vs. family member), increasing the importance of discussing patient values and social structure. Physicians should routinely conduct ACP conversations with patients with SUDs, especially those with chronic, progressive medical conditions and/or severe, uncontrolled substance use disorders.

KEY WORDS: advance care planning; substance use disorders (SUDs); medical decision-making; addiction; advance directives.

J Gen Intern Med 34(11):2630-3

DOI: $10.1007 / \mathrm{s} 11606-019-05223-\mathrm{Z}$

(c) Society of General Internal Medicine 2019

\section{INTRODUCTION}

$\mathrm{AL}$ is a 53-year-old male with a history of intravenous heroin use, major depressive disorder, panic disorder, hypertension, and tobacco use who you see in your ambulatory practice. He follows up intermittently but has been seen in the emergency department twice over the last year for accidental overdoses requiring naloxone. His wife also has an opioid use disorder

Received August 6, 2018

Revised December 28, 2018

Accepted June 28, 2019

Published online August 5, 2019 and was hospitalized last year for an overdose. AL is has been estranged from his parents and siblings for over 5 years. He has one son who is 17 years old and lives across the country. $\mathrm{AL}$ is currently living with a friend and not in contact with his wife, though they are still married. He has been reluctant to start medication-assisted therapy for opioid use disorder, though does take medication for depression. Your usual office visits normally focus on managing his medications and counseling on smoking cessation. Is this a patient who would benefit from advance care planning (ACP)?

Most primary care physicians are familiar with patients like AL. It is estimated that approximately $8 \%$ of individuals 12 years of age and older have a substance use disorder (SUD). ${ }^{1}$ SUD is defined by the DSM-V as "a condition in which the use of one or more substances leads to clinically significant impairment or distress". ${ }^{2}$ It accounts for a substantial number of emergency department visits and is associated with a multifactorial increase in morbidity and mortality. ${ }^{3-6}$ Those with SUDs have significantly higher rate of accidents, overdose, and trauma. ${ }^{3,7-12}$ Since 1999 , overdose deaths have nearly tripled, particularly from intravenous heroin and synthetic opioids such as fentanyl. ${ }^{13}$ In addition, mortality from liver disease, cardiovascular disease, cancer, infectious diseases, smoking-related conditions, and suicide is significantly higher in persons with SUDs. ${ }^{3-6,10-12,14-18}$ Finally, SUDs are more common in marginalized communities with lower average education level and socioeconomic status, which also impacts mortality. ${ }^{14,}{ }^{19}$ By and large, with the exception of driving-related accidents, mortality remains increased even in patients who are currently on medication-assisted therapy for SUDs. ${ }^{10,20}$

Mortality aside, similar to AL, patients with SUDs frequently come from families where SUDs are present through multiple generations. ${ }^{21,22}$ These family dynamics may lead to strained and estranged relationships due to economic and psychological consequences of substance use. ${ }^{23}$ By default, the law generally determines health surrogate to be spouse, followed by adult children, followed by parents, unless the patient has documented other choices. Patients with SUDs may feel closer to non-family members when considering the best individual to be their surrogate decision-maker.

Despite the increased mortality and social circumstances of people with SUDs, there has been minimal discussion on ACP 
in this population. ACP involves discussions between patients and healthcare providers about end-of-life care, ${ }^{24}$ particularly in the circumstance in which patients are unable to make decisions. SUDs are not included in any ACP guidelines nor have there been studies evaluating patterns of ACP in people with SUDs. ${ }^{24,}{ }^{25}$ We propose that there should be more discussion in the medical community about ACP in patients with SUDs.

\section{CURRENT IMPLEMENTATION OF ADVANCE CARE PLANNING}

Advance care planning (ACP) has been associated with positive outcomes including better quality of end-of-life, care more consistent with patient preferences, and improved bereavement outcomes for families. ${ }^{25}$ It is best discussed when a patient is able to participate and can be distinguished from a goals of care conversation that occurs during an acute illness. The prevalence of ACP in the primary care setting ranges from 20 to $80 \%$ when reported by physicians and from 15 to $35 \%$ when reported by patients, the higher percentages representing those with terminal illnesses. ${ }^{26}$ While there have not been studies among patients with SUDs, there has been research on ACP among other vulnerable populations, including those who are homeless, persons living with HIV, and patients with mental health disorders. Lower socioeconomic status, lower education level, social isolation, homelessness, and ethnic/ racial minorities were associated with a lower level of ACP completion. ${ }^{27-30}$ As such, we suspect that the level of ACP is also low in patients with SUDs.

This low completion of ACP is likely largely due to difficulties establishing sustainable continuing care for patients with SUDs. Multiple studies have documented difficulty with continuity of care among this population both immediately after detoxification and longer term. ${ }^{31-35}$ Often, patients with SUDs are intermittently unemployed, reducing the likelihood that they will have employer-sponsored health insurance and requiring complex navigation of the healthcare system to obtain coverage. Their coverage is often fragmented between substance use treatment centers, urgent cares, and psychiatric settings, ${ }^{36}$ where it may be less likely that providers are trained in ACP.

\section{CURRENT ACP GUIDELINES-HOW CAN SUD FIT IN?}

It has not been until fairly recently that guidelines have been developed for how to discuss ACP with patients. In 2009, the British Geriatrics Society and the Royal College of Physicians described their concise, evidence-based guidelines to ACP which recommend initiating discussions of ACP in patients with long-term conditions or those receiving end-of-life care. This society recommended initiating ACP in the outpatient setting before individuals become acutely unwell. ${ }^{24}$ More recently, the American College of Physicians published a position statement ${ }^{25}$ describing a similar subset of patients in whom ACP discussions should take place, including all patients with a serious and life-threatening illness such as metastatic cancer, congestive heart failure, chronic kidney disease, and COPD, and any patient a physician would say "no" to the question "would you be surprised if this patient died in the next year ${ }^{25}$ ?"

The term "serious and/or life-threatening illness" is traditionally applied to patients with multiple medical comorbidities. Physicians may forget that SUDs are also diseases with a chronic course involving relapse and remission and can be mild, moderate, or severe. ${ }^{37}$ One literature review noted little evidence for short-term detoxification or stabilization of SUDs; rather, recommended management of these illnesses includes screening, long-term treatment, and monitoring. ${ }^{38}$ Primary care physicians should be screening for and openly discussing SUD just as they do with diabetes, heart failure, and malignancy. And just as we encourage a patient with advanced cancer to simultaneously treat their cancer and complete ACP, we can continue to treat SUDs via medication-assisted therapy or harm reduction ${ }^{37}$ while also encouraging ACP. A phrase we use commonly at our institution is "hope for and encourage the best, but prepare for the worst."

The question "would you be surprised if this patient died within the next year" is a validated question regarding physicians' ability to predict patient mortality. ${ }^{39-41}$ In our introductory case, we described a patient, AL, who has had two overdoses in the past year. It would be difficult to find a physician who would not be surprised if AL died in the next year, yet the current guidelines do not include him. AL certainly needs assistance with treatment of his SUD or harm reduction including naloxone and clean needles if he is not ready for treatment, but, even with treatment, AL remains at high risk for mortality. His opioid use disorder also interferes with adherence to treatment for diabetes, hypertension, mental health conditions, and nicotine use, increasing his risk of an early death from heart disease, stroke, suicide, and other conditions.

\section{NEXT STEPS: WHAT CAN YOU DO?}

Programs that serve individuals with SUDs should integrate ACP into their standard practices and train clinicians on how to have ACP conversations. While ACP should ideally be incorporated into routine care of all patients with SUDs, this may not be practical. There are two validated tools through the VA that are used extensively for risk stratification of patients on chronic opioid therapy that may be applicable to patients with SUDs: (1) the Risk Index for Serious Prescription Opioid-Induced Respiratory Depression or Overdose (RIOSORD) $)^{42}$ and (2) the Stratification Tool for Opioid Risk Mitigation (STORM) ${ }^{42,}{ }^{43}$ These have been validated in large Veteran populations for predicting overdose and adverse events among patients on chronic opioid therapy. While we are mainly discussing patients with diagnosed SUDs rather 
than the larger population on chronic opioid therapy, these tools still apply. Each tool incorporates factors that have been well documented to increase the risk of overdose including a psychiatric diagnosis, a history of cerebrovascular disease, previous overdose or suicide attempt, and co-prescription of other sedating medications. ${ }^{42,}{ }^{43}$ There is also a prognostic model for overdoses among patients on chronic opioid therapy being developed in a non-VA population that found similar predictors of overdose with the addition of tobacco use and hepatitis $\mathrm{C}$ diagnoses as possible predictors. ${ }^{44} \mathrm{We}$ recommend prioritizing ACP for individuals who meet these criteria or are at an increased risk of mortality independent of substance use for reasons such as age or poorly controlled comorbidities.

With regard to addressing ACP, strong patient-provider relationships are key, particularly among those in marginalized populations. ${ }^{27}$ The specifics of ACP vary, but documenting a surrogate decision-maker for every patient is a crucial first step and should be frequently readdressed. How to do so legally will vary state by state. The next step is to discuss and document patient values and encourage completion of an advance directive that outlines acceptance and refusal of specific treatments in a future clinical scenario. ${ }^{24}$ REMAP is an acronym developed for goals of care conversations that can be adapted for $\mathrm{ACP}^{45}$ :

1. R: Raise the issue, normalize ACP, and ask permission: "I talk to all of my patients about ACP, would it be okay if we talked about this today?"

2. E: Empathize: "I know these things can be hard to talk about."

3. M: Map out what's important to the patient:

(a) Obtain a surrogate decision-maker: "Is there someone in your life you would trust to make medical decisions for you if you were unable to do so?" It is important to emphasize that this person can be family, a friend, or even a neighbor.

(b) Map out patient values using questions such as "If you were seriously ill, what would be most important to you?", and "What would you be worried about?"

4. A: Affirm the patient: "Thank you for having this discussion."

5. P: Propose a plan. This involves encouraging the patient to complete a written advance directive and have a discussion with his or her surrogate.

After the initial conversation, future visits with the patient should involve confirming the surrogate and checking in for any new thoughts if their medical condition has changed.

Many challenges may come up when discussing ACP with individuals with SUDs. This population is often not engaged with the medical system, may not be adherent to physician recommendations, and may intermittently lack capacity to make decisions. ${ }^{46}$ In general, it is difficult for patients in active substance use to think long-term, partly due to their disease and partly because they are more focused on obtaining a substance to avoid withdrawal. Other common barriers raised include time and difficulty prioritizing ACP in the setting of multiple uncontrolled comorbidities. ${ }^{47} \mathrm{We}$ also emphasize that $\mathrm{ACP}$ is not a stand-alone process, but rather part of a broader conversation with patients about their substance use. We encourage providers to incorporate ACP into discussions they are already having with patients about the risks of their substance use, creation of a safety plan, medication-assisted therapy if applicable, and care coordination.

Similar to other illnesses seen in primary care, SUDs are chronic, relapsing diseases that are difficult to treat. Patients with SUDs should be explicitly included in guidelines for ACP. Research must be done quantifying ACP in patients with SUDs and determining how best to address ACP with this population. Most physicians have seen patients with SUDs whom they would not be surprised to die in the next year, whether directly related to the SUD or not. While we work towards treating SUDs, and hope for the best, we should not forget to guide patients and their loved ones to prepare for the worst.

Corresponding Author: Michelle J. Fleshner, MD, MPH; Division of General Internal MedicineUniversity of Pittsburgh Medical Center, Pittsburgh, USA (e-mail: Fleshnerm@upmc.edu).

\section{Compliance with Ethical Standards:}

Conflict of Interest: The authors declare that they do not have a conflict of interest.

\section{REFERENCES}

1. Hunter D, Tice P. 2015 National Survey on Drug Use and Health: Methodological summary and definitions.: Center for Behavioral Health Statistics and Quality, 2016.

2. Association AP. Diagnostic and statistical manual of mental disorders (5th ed.). Arlington, VA: American Psychiatric Publishing, 2013.

3. Roerecke M, Rehm J. Cause-specific mortality risk in alcohol use disorder treatment patients: a systematic review and meta-analysis. Int $\mathrm{J}$ Epidemiol. 2014;43(3):906-19

4. Degenhardt L, Bucello C, Mathers B, et al. Mortality among regular or dependent users of heroin and other opioids: a systematic review and meta-analysis of cohort studies. Addiction. 2011;106(1):32-51

5. Degenhardt $\mathbf{L}$, Singleton $\mathbf{J}$, Calabria $\mathbf{B}$, et al. Mortality among cocaine users: a systematic review of cohort studies. Drug Alcohol Depend. 2011;113(2-3):88-95

6. Singleton $\mathbf{J}$, Degenhardt $\mathbf{L}$, Hall $\mathbf{W}$, Zabransky $\mathbf{T}$. Mortality among amphetamine users: a systematic review of cohort studies. Drug Alcohol Depend. 2009;105(1-2):1-8

7. Reece AS. Experience of road and other trauma by the opiate dependent patient: a survey report. Subst Abuse Treat Prev Policy. 2008;3:10 https://doi.org/10.1186/1747-597X-3-10 [published Online First: Epub Date].

8. Skurtveit S, Christophersen AS, Grung M, Morland J. Increased mortality among previously apprehended drunken and drugged drivers. Drug Alcohol Depend. 2002;68(2): 143-50

9. Baewert A, Gombas W, Schindler SD, et al. Influence of peak and trough levels of opioid maintenance therapy on driving aptitude. Eur Addict Res. 2007;13(3):127-35 https://doi.org/10.1159/000101548 [published Online First: Epub Date].

10. Degenhardt L, Randall D, Hall W, Law M, Butler T, Burns L. Mortality among clients of a state-wide opioid pharmacotherapy program over 20 
years: risk factors and lives saved. . Drug and Alcohol Dependence 2009; 105(1-2):9-15

11. Bardazzi G, Zanna I, Ceroti M, et al. A 5-Year Follow-Up of a Cohort of Italian Alcoholics: Hospital Admissions and Overall Survival. Alcohol Clin Exp Res. 2017;41(7):1309-18 https://doi.org/10.1111/acer.13404 [published Online First: Epub Date].

12. Raimondo Maria Pavarin AF. Mortality trends among cocaine users treated between 1989 and 2013 in Northern Italy: Results of a longitudinal study. J Psychoactive Drugs. 2017:1-9

13. Rudd RA, Seth P, David F, Scholl L. Increases in Drug and OpioidInvolved Overdose Deaths-United States, 2010-2015. MMWR Morb Mortal Wkly Rep. 2016;65:1445-52

14. Pavarin RM, Fioritti A, Sanchini S. Mortality trends among heroin users treated between 1975 and 2013 in Northern Italy: Results of a longitudinal study. J Subst Abus Treat. 2017;77:166-73

15. Hser YI, Mooney LJ, Saxon AJ, et al. High Mortality Among Patients with Opioid use disorder in a large healthcare system. J Addict Med. 2017;11(4):315-19

16. Schwarzinger M, Thiebaut SP, Baillot S, Mallet V, Rehm J. Alcohol use disorders and associated chronic disease - a national retrospective cohort study from France. BMC Public Health. 2017;18(1):43

17. Bohnert KM, Ilgen MA, Louzon S, McCarthy JF, Katz IR. Substance use disorders and the risk of suicide mortality among men and women in the US Veterans Health Administration. Addiction 2017;112(7):1193-201

18. Callaghan RC, Gatley JM, Sykes J, Taylor L. The prominence of smoking-related mortality among individuals with alcohol- or drug-use disorders. Drug Alcohol Rev 2018;37(1):97-105 https://doi.org/10 1111/dar. 12475 [published Online First: Epub Date].

19. Ghoreishi SMS, Shahbazi F, Mirtorabi SD, Ghadirzadeh MR, Hashemi Nazari SS. Epidemiological Study of Mortality Rate from Alcohol and Illicit Drug Abuse in Iran. J Res Health Sci. 2017;17(4):e00395

20. Ledberg A. Mortality related to methadone maintenance treatment in Stolkholm, Sweden, during 2006-2013. J Subst Abus Treat. 2017;74:3541

21. Reed B, Butelman ER, Yuferov V, Randesi M, Kreek MJ. Genetics of opiate addiction. Curr Psychiatry Rep. 2014;16(11):504 https://doi.org/ 10.1007/s11920-014-0504-6 [published Online First: Epub Date].

22. Yule AM, Wilens TE, Martelon MK, Simon A, Biederman J. Does exposure to parental substance use disorders increase substance use disorder risk in offspring? A 5-year follow-up study. Am J Addict. 2013;22(5):460-65

23. Substance Abuse Treatment and Family Therapy. Rockville (MD), 2004.

24. Conroy S, Fade P, Fraser A, Schiff R, Guideline Development G Advance care planning: concise evidence-based guidelines. Clin Med (Lond) 2009;9(1):76-9

25. Bernacki RE, Block SD, American College of Physicians High Value Care Task F. Communication about serious illness care goals: a review and synthesis of best practices. JAMA Intern Med. 2014;174(12):1994-2003 https://doi.org/10.1001/jamainternmed.2014.5271 [published Online First: Epub Date].

26. Glaudemans JJ, Moll van Charante EP, Willems DL. Advance care planning in primary care, only for severely ill patients? A structured review. Fam Pract. 2015;32(1):16-26 https://doi.org/10.1093/fampra/ cmu074 [published Online First: Epub Date].

27. Hubbell SA. Advance care planning with individuals experiencing homelessness: Literature review and recommendations for public health practice. Public Health Nurs. 2017;34(5):472-78 https://doi.org/10 1111/phn. 12333 [published Online First: Epub Date]

28. Leung AK, Nayyar D, Sachdeva M, Song J, Hwang Sw. Chronically homeless persons' participation in an advance directive intervention: A cohort study. Palliat Med. 2015;29(8):746-55 https://doi.org/10.1177/ 0269216315575679 [published Online First: Epub Date].

29. Sangarlangkarn A, Merlin JS, Tucker RO, Kelley AS. Advance Care Planning and HIV Infection in the Era of Antiretroviral Therapy: A Review. Top Antivir Med. 2016;23(5):174-80

30. Pellowski JA, Kalichman SC, Matthews KA, Adler N. A pandemic of the poor: social disadvantage and the U.S. HIV epidemic. Am Psychol 2013;68(4):197-209 https://doi.org/10.1037/a0032694 [published Online First: Epub Date]

31. Lee MT, Horgan CM, Garnick DW, et al. A performance measure for continuity of care after detoxification: relationship with outcomes. J
Subst Abuse Treat. 2014;47(2):130-9 https://doi.org/10.1016/j.jsat. 2014.04.002 [published Online First: Epub Date].

32. Shaefer JA, Ingudomnukul EB, Harris AH, Cronkite CR. Continuity of care practices and substance use disorder patients' engagement in continuing care. Med Care. 2005;43(12):1234-41

33. Ford LK, Zarate P. Closing the gaps: the impact of inpatient detoxification and continuity of care on client outcomes. J Psychoactive Drugs. 2010; Suppl 6:303-14

34. McLellan AT, Weinstein RL, Shen $\mathbf{Q}$, Kendig C, Levine M. Improving continuity of care in a public addiction treatment system with clinical case management. Am J Addict. 2005; 14(5):426-40 https://doi.org/10. 1080/10550490500247099 [published Online First: Epub Date].

35. Stein BD, Kogan JN, Sorbero M. Substance abuse detoxification and residential treatment among Medicaid-enrolled adults: rates and duration of subsequent treatment. Drug Alcohol Depend. 2009;104(1-2):1006 https://doi.org/10.1016/j.drugalcdep.2009.04.008 [published Online First: Epub Date].

36. Croft B, Parish SL. Care integration in the Patient Protection and Affordable Care Act: implications for behavioral health. Adm Policy Ment Health. 2013;40(4):258-63 https://doi.org/10.1007/s10488-012-04050 [published Online First: Epub Date].

37. Kampman $\mathbf{K}$, Jarvis M. American Society of Addiction Medicine (ASAM) National Practice Guideline for the Use of Medications in the Treatment of Addiction Involving Opioid Use. J Addict Med. 2015;9(5):358-67 https:// doi.org/10.1097/ADM.0000000000000166 [published Online First: Epub Date].

38. McLellan AT, Lewis DC, O'Brien CP, Kleber HD. Drug dependence, a chronic medical illness: implications for treatment, insurance, and outcomes evaluation. JAMA 2000;284(13):1689-95

39. Moss AH, Lunney JR, Culp S, et al. Prognostic significance of the "surprise" question in cancer patients. J Palliat Med. 2010;13(7):837-40 https://doi.org/10.1089/jpm.2010.0018 [published Online First: Epub Date].

40. Pang WF, Kwan BC, Chow KM, Leung CB, Li PK, Szeto CC. Predicting 12-month mortality for peritoneal dialysis patients using the "surprise" question. Perit Dial Int. 2013;33(1):60-6 https://doi.org/10.3747/pdi. 2011.00204 [published Online First: Epub Date].

41. Rhee J, Clayton JM. The 'surprise' question may improve the accuracy of GPs in identifying death in patients with advanced stage IV solid-cell cancer. Evid Based Med. 2015;20(2):71 https://doi.org/10.1136/ebmed2014-110114 [published Online First: Epub Date].

42. Zedler BK, Saunders WB, Joyce AR, Vick CC, Murrelle EL. Validation of a Screening Risk Index for Serious Prescription Opioid-Induced Respiratory Depression or Overdose in a US Commercial Health Plan Claims Database. Pain Med. 2018;19(1):68-78 https://doi.org/10.1093/ pm/pnx009 [published Online First: Epub Date].

43. Oliva EM, Bowe T, Tavakoli S, et al. Development and applications of the Veterans Health Administration's Stratification Tool for Opioid Risk Mitigation (STORM) to improve opioid safety and prevent overdose and suicide. Psychol Serv. 2017;14(1):34-49 https://doi.org/10.1037/ ser0000099 [published Online First: Epub Date].

44. Glanz JM, Narwaney KJ, Mueller SR, et al. Prediction Model for TwoYear Risk of Opioid Overdose Among Patients Prescribed Chronic Opioid Therapy. J Gen Intern Med. 2018;33(10):1646-53 https://doi.org/10. 1007/s11606-017-4288-3 [published Online First: Epub Date].

45. Childers JW, Back AL, Tulsky JA, Arnold RM. REMAP: A Framework for Goals of Care Conversations. J Oncol Pract. 2017;13(10):e844-e50 https://doi.org/10.1200/JOP.2016.018796 [published Online First: Epub Date].

46. Bauer RL. Ethical considerations regarding end-of-life planning and palliative care needs in patients with chronic psychiatric disorders. Am J Psychiatry, Residents' J 2017;11(5):4-6

47. Ramsaroop SD, Reid MC, Adelman RD. Completing an advance directive in the primary care setting: what do we need for success? J Am Geriatr Soc. 2007;55(2):277-83 https://doi.org/10.1111/j.15325415.2007.01065.x [published Online First: Epub Date]

Publisher's Note Springer Nature remains neutral with regard to jurisdictional claims in published maps and institutional affiliations. 